\title{
Native Nephrectomy in Relation to Pediatric Renal Transplantation
}

\author{
Mohammed Said ElSheemy* \\ Department of Urology, Kasr Al-Ainy hospitals, Egypt
}

Submission: November 11, 2016; Published: December 12, 2016

*Corresponding author: Mohammed Said ElSheemy, Urology department, Kasr Al-Ainy hospitals, Cairo University, Cairo, Egypt, Tel: 0201006117755; Fax: 0020235714275; Email: mohammedshemy@yahoo.com

\section{Summary}

Nowadays, renal transplantation (RTx) is the best renal replacement therapy for children with end stage renal disease (ESRD) with the best survival. However, RTx in children is more challenging than adults with differences in many aspects including the etiology, the low circulatory volume and the more difficult maintenance of the extracellular fluid volume. In many instances, the native kidneys express a significant risk to the future renal graft. Consequently, native nephrectomy (NN) is indicated in these cases. This will be associated with more challenges related to the fluid management and nutrition in these children. This article will review the issue of NN in relation to pediatric RTx including its indications, its subsequent effect in children prepared for RTx, the proper timing for NN and precautions.

The most common indications for NN are the presence of recurrent urinary tract infection (UTI) or proteinuria. Most of the diseases associated with these indications are more in children when compared with adults with ESRD due to the higher incidence of urologic anomalies and glomerulopathies. Although NN is associated with a reduction of the risk to the future renal graft as regard UTI and renal perfusion, it must be done with certain precautions to avoid any hazards to the child especially the risks resulting from loss of native urine production. Thus, timing of NN is very critical especially when bilateral NN is indicated. Medical treatment should be tried at first and if the condition is still refractory to treatment, a unilateral NN may be tried initially to keep at least one kidney as possible. Preservation of the native ureter is sometimes vital for the future possible ureteroureteral anasomosis or ureterocystoplasty particularly when facing post RTx ureteric complications.

Keywords: Native nephrectomy; Pediatric renal transplantation; Proteinuria; Polyurea; Ureterocystoplasty; Ureteroureteral anastomosis

Abbreviations: ARPKD: Autosomal Recessive Polycystic Kidney Disease; CIC: Clean Intermittent Catheterization; ESRD: End Stage Renal Disease; FSGS: Focal Segmental Glomeruloscelerosis; GFR: Glomerular Filtration Rate; NN: Native Nephrectomy; PUV: Posterior Urethral Valves; RTx: Renal Transplantation; VUR: Vesicoureteric Reflux; UTI: Urinary Tract Infection

\section{Introduction}

Nowadays, renal transplantation (RTx) is the best renal replacement therapy for children with end stage renal disease (ESRD) with the best survival [1]. However, RTx in children is more challenging than adults with differences in many aspects including the etiology of ESRD, the low circulatory volume and the more difficult maintenance of the extracellular fluid volume [2-3]. In many instances, the native kidneys express a significant risk to the future renal graft. Consequently, native nephrectomy (NN) is indicated in these cases. This will be associated with more challenges related to the fluid management and nutrition in these children. This article will review the different indications for NN, its subsequent effect in children prepared for RTx and the proper timing for nephrectomy in relation to RTx. The items covered in this mini-review are presented in Table 1.
Table 1: Items covered in this minireview.

\begin{tabular}{|c|}
\hline Indications for native nephrectomy and reported advantages \\
\hline Is it important to preserve the ureter? \\
\hline Ureterocystoplasty \\
\hline Ureteroureteral anastomosis \\
\hline Timing of native nephrectomy in relation to peditric renal \\
transplantation \\
\hline
\end{tabular}

\section{Indications for native nephrectomy}

NN may be required in $20-45 \%$ of pediatric recipients [25]. The most common indications are the presence of heavy proteinuria $(>40 \mathrm{mg} / \mathrm{m} 2 / \mathrm{h})$ or chronic renal parenchymal infection with associated highgrade vesicoureteric reflux (VUR), hydronephrosis or calculi. Other causes include polyuria $(>2.5$ $\mathrm{ml} / \mathrm{kg} / \mathrm{h}$ ), severe uncontrollable hypertension related to the 
native kidney or polycystic disease causing symptoms arising from extremely large size, pain, hematuria or pressure effects causing gastrointestinal or respiratory symptoms (Table 2) [2-8]. Most of these indications are more common in children with ESRD as the congenital urologic abnormalities and glomerulopathies predominate in children when compared to adults $[1,9]$. As a result, the issue of NN is more important in pediatric patients with ESRD.

Table 2: Indications for native nephrectomy [4-12].

\begin{tabular}{|l|}
\hline 1- Recurrent renal parenchymal infection: \\
\hline Infected hydronephrosis \\
\hline Infected reflux \\
\hline Infected urolithiasis \\
\hline 2- Drug resistant proteinuria \\
\hline 3- Refractory hypertension \\
\hline 4- Polyuria \\
\hline 5- Combined indications: \\
\hline Polycystic disease \\
\hline
\end{tabular}

In our center, the most common causes of NN were proteinuria $(32 \%)$ and urinary tract infection (UTI) in abnormal urinary tract $(15 \%)$. Totally, NN was required in $46.6 \%$ of children [3]. Fraser, et al. reported 21/203 (10.3\%) children listed for RTx underwent $32 \mathrm{NN}$. Indications were drug-resistant proteinuria in six children, recurrent upper tract urosepsis in another six children, refractory hypertension in four children, malignancy/ malignant predisposition in four children and concomitant procedure during ureterocystoplasty in one child [8].

\section{Proteinuria}

Table 3: Consequences of heavy proteinuria [6,17-24].

\begin{tabular}{|l|}
\hline 1- Hypoalbuminemia \\
\hline 2- Nutritional deficit \\
\hline 3- Delayed wound healing. \\
\hline 4- Intravascular volume depletion. \\
\hline 5- Graft hypoperfusion \\
\hline $\begin{array}{l}\text { 6- Loss of anti-thrombotic factors in the urine with resultant disturbed } \\
\text { hemostasis }\end{array}$ \\
\hline 7- Thromboembolic events and graft thrombosis. \\
\hline
\end{tabular}

Many causes of ESRD are associated with loss of proteins in urine. When the proteinuria is massive, it will be associated with potential risks including intravascular volume depletion, nutritional deficit, and delayed wound healing. Intravascular volume depletion may lead to graft hypoperfusion especially during transplantation and the early postoperative period $[4,10,11]$. Additionally, heavy proteinuria and hypoalbuminemia may increase the risk of thromboembolic events [11,12]. This is well reported in nephrotic syndrome and other diseases like focal segmental glomeruloscelerosis (FSGS) due to the associated loss of anti-thrombotic factors in the urine with resultant disturbed hemostasis (Table 3) [11-13]. Consequently, removing the native kidney in patients with heavy proteinuria is expected to reduce all these risks including the risk of acute graft thrombosis Fraser, et al. $[8,10,11]$ reported six children listed for RTx underwent pre-RTx NN for persistent heavy proteinuria; three had congenital nephrotic syndrome and were dependent on albumin infusions, two had FSGS, and one had HenocheSchonlein purpura. The six children had significant protein losses despite medical treatment in the form of corticosteroids, second-line cytotoxic therapy, ACE inhibitors and loop diuretics. Out of the six children who had initially undergone unilateral nephrectomy, three required further nephrectomy due to significant ongoing proteinuria, on average 1 year later. Unilateral nephrectomy was sufficient to reduce the proteinuria to acceptable levels in only two children, one with FSGS and one with congenital nephrotic syndrome [8]. Ghane documented modest rise of serum proteins after unilateral nephrectomy and full correction of serum albumin, total proteins and fibrinogen concentrations after bilateral nephrectomy in their study on 22 hypoalbuminemic patients prepared for RTx.

Glomerular diseases especially FSGS were the cause in 50\% and cystinosis in another $32 \%$ while congenital abnormalities of the urinary tract were present in $14 \%$ of these patients. Proteinuria decreased from 157 to $100 \mathrm{mg} / \mathrm{m} 2 / \mathrm{h}(-40 \%)$ after unilateral nephrectomy. Within 2 months post bilateral nephrectomy, initially low albumin and total serum protein concentrations increased by 33 and $17 \%$, respectively, while previously elevated fibrinogen concentrations decreased by $42 \%$ to almost normal levels. Serum albumin, protein and fibrinogen concentrations normalized in 93,73 , and $55 \%$ of nephrectomized patients, respectively [4]. Medical treatment is usually preferred before the decision of NN which is taken in cases with refractory massive proteinuria [9]. Some authors tried medications with only one side nehrectomized leaving the contralateral side to be done with RTX $[8,14]$. The original cause of ESRD may sometimes recur in the renal graft. Consequently, some authors perform native nephrectomy in these recurrent diseases like FSGS to improve the ability to diagnose disease recurrence early, which may lead to more effective treatment [11].

\section{Polyuria}

Many causes of ESRD are associated with polyuria especially in the presence of tubular disorders or renal dysplasia. These children may continue to excrete large amounts of native urine output for prolonged periods after RTx. The risk of graft hypoperfusin is increased in these children due to massive polyuria and extracellular volume depletion especially if associated with perfusion mismatch between these small recipients and adult donors [4]. These risks are exacerbated by the fact that the young recipient may excrete a volume of urine every hour that approximates to his total blood volume. As a result, even a small error in replacement therapy may create a major fluid and electrolyte imbalance [15]. Graft hypoperfusion may be complicated by tubular necrosis or graft thrombosis while in 
chronic cases, irreversible drop in glomerular filtration rate (GFR) and accelerated chronic graft fibrosis may occur particularly in younger children $[10,16,17]$. Naesen, et al. reported progressive non-immune graft injury in children after receiving adultsize grafts. They postulated histologically that chronic tubulointerstitial damage is predisposed by graft ischemia associated with donor-recipient size discrepancy [17]. In a later study using the cDNA microarray techniques, they reported that early graft hypoperfusion induced expression of profibrotic genes which was evident before any histological appearance of fibrosis [18]. Volume depletion and renal hypoperfusion are associate also with other risks including exacerbation of drug nephrotoxicity including immunosuppressive drugs (Table 4) [19]. NN in these children particularly if polyuria is $>2.5 \mathrm{ml} / \mathrm{kg} / \mathrm{h}$ will reduce the risk of post-RTx graft hypoperfusion particularly if the recipient and the parents cannot cope with the high requirements of fluid intake [4]. On the other hand, many polyuric children become oliguric after a period on dialysis and so unilateral nephrectomy or even no nephrectomy may be appropriate, providing there are normal levels of circulating antithrombotic factors and near normal plasma albumin levels [8].

Table 4: Consequences of massive plyuria $[6,17,27,29-38]$.

\begin{tabular}{|l|}
\hline 1- Intravascular volume depletion. \\
\hline 2- Graft hypoperfusion \\
\hline 3- Tubular necrosis \\
\hline 4- Graft thrombosis \\
\hline 5- Irreversible drop in glomerular filtration rate \\
\hline 6- Accelerated chronic graft fibrosis may \\
\hline $\begin{array}{l}\text { 7- Exacerbation of drug nephrotoxicity including immunosuppressive } \\
\text { drugs }\end{array}$ \\
\hline
\end{tabular}

\section{Hypertension}

Hypertension is common in patients with chronic kidney disease and may be caused by fluid overload or by increased renin secretion [20]. Cavallini, et al. [21] compared retrospectively young adults who had undergone RTx in the pediatric age (with a mean follow-up of 10 years) with or without NN for hypertension. Fourteen patients had undergone a bilateral pre-RTx NN, 18 had undergone a unilateral NN, mostly at the time of transplantation and the native kidneys were left in place in the remaining 35 patients. Patients with more severe hypertension were more likely to have undergone NN. The number of antihypertensive drugs used prior to transplantation was significantly higher in the nephrectomized groups. At follow-up, the amount of antihypertensive medications was similar between groups and no significant differences were observed in mean arterial blood pressure [21]. This reduction in the number of antihypertensive drugs was reported also in other studies [22]. Fraser, et al [8] reported four children listed for RTx underwent NN for refractory hypertension. Out of the four children, three underwent synchronous bilateral NN. Two children had bilateral dysplastic kidneys with dilatation, and one had autosomal recessive polycystic kidney disease (ARPKD) and one FSGS. Two had presented with hypertensive encephalopathy. All were on maximal multiagent anti-hypertensive therapy [8].

\section{Recurrent urinary tract infection}

Indications for pre-RTx NN include chronic renal parenchymal infection with associated highgrade VUR, hydronephrosis or calculi [5,7]. Infections are major complication post-RTx. The most common form of bacterial infection is UTI [23]. Some authors found a significant decrease of GFR at the time of UTI, indicating parenchymal involvement [24]. However, no sustained deterioration of renal function was detected. Other authors reported significantly lower GFR in patients with recurrent UTI with long-term affection of the graft function [25]. Fraser, et al [8] reported six children listed for RTx who underwent NN for significant symptomatic recurrent upper tract infections despite antibiotic prophylaxis. Most had underlying obstructive uropathy and VUR. One child had undergone previous ureteric reimplantation [8]. In another study on 195 RTx in children, bilateral nephroureterectomies were performed in two children with meningomyelocele and six children with posterior urethral valve (PUV), because of the presence of grade III to IV VUR and/or significant hydroureteronephrosis. In two children, nephroureterectomy was performed at the time of RTx. It was conducted as a staged procedure in the remainder. Unilateral NN because of infected hydronephrosis or reflux was performed in three other children [26].

\section{Polycystic disease}

Indications for $\mathrm{NN}$ prior to renal transplantation in patients with ARPKD include extremely large kidneys with pressure effects (gastrointestinal or respiratory symptoms) or reducing the available space for future graft, pain, hematuria or hypertension [5,7]. The renin-angiotensin system is believed to account for the hypertension observed among these patients [20]. Bergmann, et al. [27] showed that $86 \%$ of the children with ARPKD exhibit a GFR below the third centile at a median age of 4 years, $92 \%$ showed kidney length more than two standard deviations compared with the normal population and $76 \%$ showed hypertension at a median age of $13 \mathrm{yr}$, growth retardation, anemia, and evidence of portal hypertension. NN may be indicated earlier in life to allow space for dialysis or to improve respiration Fraser, et al. [8] reported bilateral NN performed for a child with ARPKD for significant, symptomatic recurrent upper tract infections despite antibiotic Prophylaxis [8].

\section{Is it important to preserve the ureter?}

When NN is indicated, the ureter is usually preserved. However, native ureterectomy should be done as a part of native nephroureterectomy in certain conditions particularly when nephrectomy is indicated for recurrent UTI with refluxing ureter and/or significant hydroureteronephrosis [26]. Otherwise, 
preservation of the native ureter has many advantages especially for restoration of the urinary tract contunuity and reconstruction of the bladdder when indicated.

\section{Ureterocystoplasty}

When bladder augmentation is indicated to optimize the intravesical pressure to receive the future renal graft, the dilated ureter provides an excellent source of augmentation material with urothelium free from electrolyte and acid base disturbances, mucous production and risk of neoplasia [29]. Thus, when NN is indicated, the native ureter should be preserved if ureterocystoplasty is planned. The use of ureter to augment the bladder prior to RTx was reported in many studies [28-30]. However, a follow-up urodynamic study should be performed after ureterocystoplasty to confirm improvement of the compliance and bladder capacity, before doing RTx as the child may still need to augment the bladder using a bowel segment if the bladder conditions did not improve after ureterocystoplasty. If the ureter is not dilated or there is a history of previous ureterovesical implantation, the ureter is no longer candidate for ureterocystoplasty in these children due to impaired blood supply of the lower segment [29,30]. Nahas, et al. reviewed 211 children who underwent 226 renal transplants. A total of 15 children underwent bladder augmentation. In eight patients the bladder augmentation was initially performed with dilated ureters, with both ureters being used in two patients and a single ureteral unit being used in six. Urodynamic studies confirmed non-improved bladder capacity or compliance in two patients. They were further submitted to classic enterocystoplasty [30]. Fraser, et al. reported a one child who underwent NN with use of the native ureter simultaneously for ureterocystoplasty. This child had PUV, a small-capacity, high-pressure bladder, and bilateral VUR with gross ureteric dilatation. Unilateral NN was done and the most dilated ureter (and poorest kidney) was utilized for bladder augmentation [8].

\section{Ureteroureteral anastomosis}

Whenever possible, the ureter should be implanted in children using nonrefluxing technique [2,3,26,31]. The nonrefluxing technique is more critical in children with neobladder or augmented bladder to prevent contaminated urine from refluxing into the upper urinary tract especially if these children are on clean intermittent catheterization (CIC) as the rate of UTI is higher in these children on CIC or enterocystoplasty $[30,32]$. It was reported that the most serious complications of enterocystoplasty in renal transplant patients are infectious complications and sepsis.33 The most common technique used for non-refluxing ureterovesical anastomosis is the stented tunneled extravesical procedure (LichGregoir) [2,3,6,33,34]. However, other types are also performed including the PolitanoLeadbetter technique.6 When the bladder is augmented, it is better to reimplant the ureter into the native bladder segment in the reservoir as it is the best site for the ureteral reimplantation $[26,30]$. When this is not available due to small bladder segment, the ureter can be implanted in non-refluxing manner into the bowel segment Ali El-Dein et al. [26,30] reimplanted the ureters into the ileal part of the ileocystoplasty via nonrefluxing serous lined extramural tunnels.26 Sometimes, the ureter cannot be implanted in nonrefuxing method even to the bowel due to difficult dissection of the reservoir. In these cases, the last option for antireflux reimplantation is the ureteroureteral anastomosis to the native ureter (after nephrectomy) provided that the native ureter is non-refluxing. This was reported in many studies [26,30]. Nahas et al. tried to reimplant the ureter into the native bladder using an antireflux procedure in 19 patients with augmented bladder or urinary diversion but this was possible only in 13 patients. In the remaining patients, it was implanted into the bowel portion of the reservoir or to the native ureter.30 In the study reported by Irtan et al, ureteral anastomosis was ureterovesical in 185 cases, pyeloureteral in three, uretero-ureteral in 13 , and one was re-implanted into an ileal conduit. In the 185 ureterovesical cases, it was performed with the Lich-Gregoire technique in 183 grafts, and with the Lead-better Politano technique in two children [6]. The native ureter is also useful in transplanted children with ureteric complications including leaks from ureterovesical anastomosis or ischemic stenotic ureterovesical segment [2]. The reported post-RTx ureteric complications are $2-20 \% .35$ The anastomosis to the native ureter may be the best available option in these children $[2,30]$. Nahas, et al. reported three patients presented with necrosis of the distal portion of the ureter and were successfully treated with ureteroureterostomy using the native ureter $[30,35]$.

\section{Timing of native nephrectomy in relation to peditric RTx}

When NN is indicated, It is unclear whether unilateral nephrectomy is adequate, or if bilateral nephrectomy should be performed whether this is a synchronous or staged procedure [8]. NN is usually performed prior to RTx. Staging can be done in bilateral cases with one side performed at the time of RTx at the upper aspect of the transplant incision while the contralateral NN is performed earlier $[2,3,26]$. The timing for $\mathrm{NN}$ is a critical decision. In addition to the potential morbidity related to the incision and the increase in the number of procedures to which the child is exposed before RTx, the child may be left anuric which will make the dialysis more difficult [5]. So, postponing one side to be nephrectomized at the time of RTx; will help to preserve some urine production which can facilitate dialysis management $[4,5,36]$. Otherwise, loss of urine output may result in more difficult fluid control, fluid overload and/or volume mediated hypertension. This was reported in some studies [4,34]. Other complications of NN may occur, including peritoneal leaks due to laceration that requir temporary hemodialysis instead of the planned initiation or continuation of peritoneal dialysis [4]. Another drawback for pre-RTx NN with susequent anuria, is obligatory need for bladder cycling if bladder augmentation was done. In these children, continuous bladder irrigation is essential 
to maintain the capacity of the augmented bladder [3,34]. This is more important in children due to the high incidence of lower urinary tract dysfunctions in children with ESRD (20-30\%) with subsequent higher rate of bladder reconstruction $[1,3,37,38]$. Functional augmentation is preferable to dry augmentation because it allows development of adequate bladder capacity and evaluation of bladder continence and function before transplantation. Anuric patients undergoing cystoplasty who are scheduled to receive a graft may be hospitalized in some centers before RTx for bladder cycling and assessment of capacity prior to the transplant $[39,40]$. Other centers perform periodical hydrodistention of the augmented bladder to prevent its shrinkage [34]. The better alternative is to stage bilateral NN to give the advantage of recycling the augmented bladder by the remaining kidney in the period before transplantation [26]. Reconstruction before transplantation has also been associated with other potential problems including the accumulation of mucus or acid contents in the dry cystoplasty reservoir when performing enterocystoplasty or gastrocystoplasty in anuric patients. This may be complicated by severe irritation, infection, or bladder perforation.40 Additionally, anephric patients have a greater degree of renal anemia and more severe renal osteodystrophy from 1,25 dihydroxycholecalciferol deficiency. Preserving one native kidney may reduce these complications [8].

\section{Conclusion}

The most common indications for native nephrectomy are the presence of recurrent UTI or proteinuria. Most of the the diseases associated with these indications are more in children when compared with adults with ESRD due to the higher incidence of urologic anomalies and glomerulopathies.

Although NN is associated with a reduction of the risk to the future renal graft as regard UTI and renal perfusion, it must be done with certain precautions to avoid any hazards to the child especially the risks resulting from loss of native urine production. Thus, timing of NN is very critical especially when bilateral NN is indicated. Medical treatment should be tried at first and if the condition is still refractory to treatment, a unilateral NN may be tried initially to keep at least one kidney as possible. Preservation of the native ureter is sometimes vital for the future possible ureteroureteral anasomosis or ureterocystoplasty particularly when facing post-RTx ureteric complications.

\section{References}

1. http://www.usrds.org/2015/view/v2_08.aspx

2. ElSheemy MS, Shouman AM, Shoukry AI, Soaida S, Salah DM, et al (2014) Surgical complications and graft function following live-donor extraperitoneal renal transplantation in children $20 \mathrm{~kg}$ or less. J of Pediat Urol 10(4): 737-43.

3. Saad IR, Habib E, ElSheemy MS, Abdel-Hakim M, Sheba M, et al. (2016) Outcomes of living donor renal transplantation in children with lower urinary tract dysfunction: a comparative retrospective study. BJU Int 118(2): 320-326.
4. Sharbaf FG, Bitzan M, Szymanski KM, Bell LE, Gupta I, et al. (2012) Native nephrectomy prior to pediatric kidney transplantation: biological and clinical aspects. Pediatr Nephrol 27(7): 1179-1188.

5. CRC press (2006) Kidney transplantation. In: Docimo SG, Canning DA, Khoury AE $\left(5^{\text {th }}\right.$ edn) The Kelalis-King-Belman textbook of clinical pediatric urology, London, Taylor \& Francis, New York and Philadelphia, USA, pp. 367-385.

6. Irtan S, Maisin A, Baudouin V, Nivoche Y, Azoulay R, et al. (2010) Renal transplantation in children: critical analysis of age related surgical complications. Pediatr Transplant 14(4): 512-519.

7. EBPG Expert Group on Renal Transplantation (2002) European best practice guidelines for renal transplantation. Section IV: Long-term management of the transplant recipient. IV.11 Paediatrics (specific problems). Nephrol Dial Transplant 17(Suppl 4): 55-58.

8. Fraser N, Lyon PC, Williams AR, Christian MT, Shenoy MU (2013) Native nephrectomy in pediatric transplantation--less is more! J Pediatr Urol 9(1): 84-89.

9. Chacko B, Rajamanickam T, Neelakantan N, Tamilarasi V, John GT (2007) Pediatric renal transplantation: a single center experience of 15 yr from India. Pediatr Transplant 11(8): 844-849.

10. Harmon WE, Stablein D, Alexander SR, Tejani A (1991) Graft thrombosis in pediatric renal transplant recipients. A report of the North American Pediatric Renal Transplant Cooperative study. Transplantation 51(2): 406-412.

11. Greenstein SM, Delrio M, Evan O, Fuerstein D, Schechner R, et al. (2000) Plasmapheresis treatment for recurrent focal sclerosis in pediatric renal allografts. Pediatr Nephrol 14(12): 1061-1065.

12. Lau SO, Tkachuck JY, Hasegawa DK, Edson JR (1980) Plasminogen and antithrombin III deficiencies in the childhood nephrotic syndrome associated with plasminogenuria and antithrombinuria. J Pediatr $96(3$ Pt 1): 390-392.

13. Singhal R, Brimble KS (2006) Thromboembolic complications in the nephrotic syndrome: pathophysiology and clinical management. Thromb Res 118(3): 397-407.

14. Jalanko H (2009) Congenital nephrotic syndrome. Pediatr Nephrol 24(11): 2121-2128.

15. Rocca GD, Costa MG, Bruno K, Coccia C, Pompei L, et al. (2001) Pediatric renal transplantation: anesthesia and perioperative complications. Pediatr Surg Int 17(2-3): 175-179.

16. Salvatierra O, Singh T, Shifrin R, Conley S, Alexander S, et al. (1999) Transplantation of adult-sized kidneys into infants induces major blood flow changes. Transplant Proc 31(1-2): 236-237.

17. Naesens M, Kambham N, Concepcion W, Salvatierra O, Sarwal M (2007) The evolution of nonimmune histological injury and its clinical relevance in adult-sized kidney grafts in pediatric recipients. Am J Transplant 7(11): 2504-2514.

18. Naesens M, Khatri P, Li L, Sigdel TK, Vitalone MJ, et al. (2011) Progressive histological damage in renal allografts is associated with expression of innate and adaptive immunity genes. Kidney Int 80(12): 1364-1376.

19. Jensen CW, Jordan ML, Schneck FX, Shapiro R, Tzakis A, et al. (1991) Pediatric renal transplantation under FK 506 immunosuppression. Transplant Proc 23(6): 3075-3077.

20. Goto M, Hoxha N, Osman R, Dell KM (2010) The renin-angiotensin system and hypertension in autosomal recessive polycystic kidney disease. Pediatr. Nephrol 25(12): 2449-2457.

21. Cavallini M, Zazzo GD, Giordano U, Pongiglione G, Strologo LS, et al. (2010) Long-term cardiovascular effects of pre-transplant native kidney nephrectomy in children. Pediatr Nephrol 25(12): 2523-2529. 
22. Gawish AE, Donia F, Fathi T, Al-Mousawi M, Samhan M (2010) It takes time after bilateral nephrectomy for better control of resistant hypertension in renal transplant patients. Transplant Proc 42(5): 1682-1684.

23. Goya N, Tanabe K, Iguchi Y, Oshima T, Yagisawa T, et al. (1997) Prevalence of urinary tract infection during outpatient follow-up after renal transplantation. Infection 25(2): 101-105.

24. Sharifian M, Rees L, Trompeter RS (1998) High incidence of bacteriuria following renal transplantation in children. Nephrol Dial Transplant 13(2): 432-435.

25. Herthelius M, Oborn $H$ (2007) Urinary tract infections and bladder dysfunction after renal transplantation in children. J Urol 177(5): 1883-1886.

26. Ali-El-Dein B, Abol-Enein H, El-Husseini A, Osman Y, Shehab El-Din AB, et al. (2004) Renal Transplantation in Children With Abnormal Lower Urinary Tract. Transplant Proc 36(10): 2968-2973.

27. Bergmann C, Senderek J, Windelen E, Küpper F, Middeldorf I, et al (2005) Clinical consequences of PKHD1 mutations in 164 patients with autosomalrecessive polycystic kidney disease (ARPKD). Kidney Int 67(3): 829-848.

28. Prelog M, Bergmann C, Ausserlechner MJ, Fischer H, Margreiter R, et al. (2006) Successful transplantation in a child with rapid progression of autosomal recessive polycystic kidney disease associated with a novel mutation. Pediatr Transplant 10(3): 362-366.

29. Nahas WC, Lucon M, Mazzucchi E, Antonopoulos IM, Piovesan AC, et al. (2004) Clinical and urodynamic evaluation after ureterocystoplasty and kidney transplantation. J Urol 171(4): 1428-1431.

30. Nahas WC, Antonopoulos IM, Piovesan AC, Pereira LM, Kanashiro H, et al. (2008) Comparison of renal transplantation outcomes in children with and without bladder dysfunction. A customized approach equals the difference. J Urol 179(2): 712-716.

31. Barrero R, Fijo J, Fernandez-Hurtado M, García-Merino F, León E, et al. (2007) Vesicoureteral reflux after kidney transplantation in children. Pediatr Transplant 11(5): 498-503.
32. Neild GH, Darkmish A, Wood S, Nauth-Misir R, Woodhouse CR (2004) Renal transplantation in adults with abnormal bladders. Transplantation 77(7): 1123-1127.

33. Rischmann P, Malavaud B, Bitker MO, Chretien Y, Dawahara M, et al. (1995) Results of 51 renal transplants with the use of bowel conduits in patients with impaired bladder function: a retrospective multicenter study. Transplant Proc 27(4): 2427-2429.

34. Ghirardo G, Midrio P, Zucchetta P, Gamba P, Zanon G, et al. (2015) Renal transplantation in children weighing $<15 \mathrm{~kg}$ : does concomitant lower urinary tract dysfunction influence the outcome? Pediatr Nephrol 30(8): 1337-1342.

35. Praz V, Leisinger HJ, Pascual M, Jichlinski P (2005) Urological complications in renal transplantation from cadaveric donor grafts: a retrospective analysis of 20 years. Urol Int 75(2): 144-149.

36. Shafi T, Jaar BG, Plantinga LC, Fink NE, Sadler JH, et al. (2010) Association of residual urine output with mortality, quality of life, and inflammation in incident hemodialysis patients: the Choices for Healthy Outcomes in Caring for End-Stage Renal Disease (CHOICE) study. Am J Kidney Dis 56(2): 348-58.

37. Adams J, Mehls O, Wiesesl M (2004) Pediatric renal transplantation and the dysfunctional bladder. Transpl Int 17(10): 596-602.

38. Mendizabal S, Estornell F, Zamora I, Sabater A, Ibarra FG, et al. (2005) Renal transplantation in children with severe bladder dysfunction. J Urol 173(1): n226-229.

39. Nahas WC, David-Neto E (2009) Strategies to treat children with endstage renal dysfunction and severe lower urinary tract anomalies for receiving a kidney transplant. Pediatr Transplant 13(5): 524-535.

40. Hatch DA (1994) Kidney transplantation in patients with an abnormal lower urinary tract. Urol Clin North Am 21(2): 311-320. 